\title{
Effects of high-intensity training on MCT1, MCT4, and NBC expressions in rat skeletal muscles: influence of chronic metabolic alkalosis
}

\author{
Claire Thomas, ${ }^{1,3}$ David Bishop, ${ }^{1,4}$ Tom Moore-Morris, ${ }^{1}$ and Jacques Mercier ${ }^{1,2}$ \\ ${ }^{1}$ Université Montpellier 1, UFR Médecine EA701, F-34295, Montpellier; ${ }^{2}$ Institut National de la Santé et de la Recherche, \\ ERI 25, F-34295, Montpellier; ${ }^{3}$ Université Evry Val d'Essonne, UFR Sciences fondamentales et appliquées, EA3872, \\ Département Sciences et Techniques des Activités Physiques et Sportives, F-91025, Evry, France; and ${ }^{4}$ Facoltà di Scienze \\ Motorie, Università degli Studi di Verona, Verona, Italy
}

\begin{abstract}
This study investi- gated the effects of high-intensity training, with or without induced metabolic alkalosis, on lactate transporter (MCT1 and MCT4) and sodium bicarbonate cotransporter (NBC) content in rat skeletal mus- cles. Male Wistar rats performed highintensity training on a treadmill 5 times/wk for $5 \mathrm{wk}$, receiving either sodium bicarbonate (ALK-T) or a placebo (PLA-T) prior to each training session, and were compared with a group of control rats $(\mathrm{CON})$. MCT1, MCT4, and NBC content was measured by Western blotting in soleus and extensor digitorum longus (EDL) skeletal muscles. Citrate synthase (CS) and phospho- fructokinase (PFK) activities and muscle buffer capacity $([3 \mathrm{~m})$ were also evaluated. Following training, CS and PFK activities were significantly higher in the soleus only $(P<0.05)$, whereas $[3 \mathrm{~m}$ was significantly higher in both soleus and EDL $(P<0.05)$. MCT1 (PLA-T: 30\%; ALK-T: $23 \%$ ) and NBC contents (PLA-T: 85\%;

ALK-T: $60 \%)$ increased significantly only in the soleus following training $(P<0.01)$. MCT4 content in the soleus was significantly greater in ALK-T (115\%) but not PLA-T compared with CON. There was no significant change in protein content in the EDL. Finally, NBC content was related only to MCT1 content in soleus $(r=0.50, P<$ 0.01). In conclusion, these results suggest that MCT1, MCT4, and NBC undergo fiber-specific adaptive changes in response to highintensity training and that induced alkalosis has a positive effect on training-induced changes in MCT4 content. The correlation between MCT1 and NBC expression suggests that lactate transport may be facilitated by NBC in oxidative skeletal muscle, which may in turn favor better muscle $\mathrm{pH}$ regulation.
\end{abstract}

monocarboxylate transporter; sodium bicarbonate transporter; lactate transporter; muscle $\mathrm{pH}$ regulation; exercise

DURING HIGH-INTENSITY EXERCISE, contracting skeletal muscles produce and accumulate lactate and hydrogen ions. Lactate is either removed by oxidative processes or released into the blood and removed by other cells [i.e., the lactate shuttle (11)]. Muscle $\mathrm{pH}$ dramatically decreases during high-intensity exercise $[\mathrm{pH}=7.4=>6.86(34)]$ and then, consequently, in the blood. Efficient $\mathrm{pH}$ regulation depends on different mechanisms, including membrane transport proteins (for review, see Ref. 21), sarcolemmal carbonic anhydrase (16), and also the intracellular muscle buffer capacity (33).

Address for reprint requests and other correspondence: C. Thomas, Université d'Evry Val d'Essonne, Département STAPS, UFR des Sciences fondamentales et appliquées, Laboratoire d'Etude de la Physiologie de l'Exercice (EA 3872), Bvd François Mitterrand, 91025 Evry Cedex, France (e-mail: thomasclaire@wanadoo.fr).
The main transport proteins that regulate muscle $\mathrm{pH}$ are the sodium/proton exchanger [NHE $(19,24)]$, the monocarboxylate transporters [MCT (6)], and the sodium bicarbonate cotransporter [NBC (26)]. NHE plays an important role in $\mathrm{pH}$ regulation at rest, whereas MCTs are involved mainly during high-intensity exercise $(19,24)$. Different isoforms of MCT are expressed in skeletal muscle (8), with the most commonly described being the MCT1 and MCT4 isoforms. MCT1 has been found predominantly in oxidative muscles, and only in small amounts in glycolytic muscles, whereas MCT4 has been reported to exist with less fiber-type specificity $(14,35)$. Finally, NBC has been identified in different cells, and only recently in human $(25,26)$ and rat $(26)$ skeletal muscle. NBC and MCT1 seem to functionally cooperate with Becker et al. (3) reporting increased lactate transport activity when MCT1 is expressed together with NBC in xenopus oocytes. Facilitation of MCT1 transport activity appears due mainly to the dissipation of the proton gradient with the transport of bicarbonate by NBC.

Although many studies (21) have investigated changes in MCT relative abundance following various forms of training, there are limited data regarding the training response of NHE and no data regarding training-induced changes in NBC abundance. The expression of MCT1, but not MCT4, is responsive to endurance training $(14,34)$, whereas the expression of both isoforms is responsive to strength (23) and sprint (5) training. Thus, the type of training does not seem of importance for the regulation of MCT1 content in skeletal muscle, although it appears important for the regulation of MCT4 content (23). In contrast, both muscle buffer capacity (15) and NHE (24) have been reported to undergo only adaptive changes in response to high-intensity training. These results suggest that a reduction in internal $\mathrm{pH}$ could be an important stimulus for training-induced adaptations in the MCT1 and MCT4 protein expression and possibly also NBC expression.

The efflux of $\mathrm{H}^{+}$from the muscle can be enhanced by an elevated extracellular buffer concentration (20). The ingestion of a buffering agent [i.e., sodium bicarbonate $\left(\mathrm{NaHCO}_{3}\right)$ or sodium citrate] prior to exercise has subsequently been shown to reduce the accumulation of $\mathrm{H}^{+}$in skeletal muscle, interstitium, and blood during repeated, intense muscle contractions $(27,38)$. Although previously used as an ergogenic aid, ingestion of a blood-buffering agent provides a novel approach to alter the $\mathrm{H}^{+}$accumulation during training and to investigate 
the role of $\mathrm{H}^{+}$accumulation as a stimulus for changes in transport proteins.

The main purpose of this study, therefore, was to determine the effects of high-intensity exercise with or without the ingestion of $\mathrm{NaHCO}_{3}$ prior to each training session on MCT1, $\mathrm{MCT} 4$, and NBC protein relative abundance in rats. Due to the possible ergogenic effects of $\mathrm{NaHCO}_{3}$ ingestion, the experimental training groups were matched for work (supervised training program for $5 \mathrm{wk}$ ). We hypothesized that high-intensity exercise training would increase MCT1, MCT4, and NBC protein relative abundance in rat skeletal muscle. Furthermore, if intracellular $\mathrm{pH}$ is an important stimulus for changes in these proteins, then induced metabolic alkalosis, via the chronic ingestion of bicarbonate prior to each training session, should differently influence training-induced changes in their relative abundance. Finally, due to their functional cooperation (3) and their expression in skeletal muscle (26), we further posited that NBC and MCT1 relative abundance would be correlated.

\section{METHODS}

Animals

Experiments were conducted on 28 male Wistar rats (Charles River, St. Germain sur l'Arbresle, France) according to the guidelines of the National Research Council for the care and use of laboratory animals and French law on animal handling and protection. The protocol was approved by the local ethics committee. The rats were individually housed in a temperature-controlled room and maintained with food and drink ad libitum in a 12:12-h light-dark cycle (lights on at $7 \mathrm{PM}$ ), thus allowing exercise during their active phase. All exercise tests were conducted in the daytime, but a dark cover was placed over the treadmill to ensure that the rats exercised in darkness. The body mass of the rats was monitored throughout the experimental period.

\section{Experimental Design}

All animals were familiarized with a motor-driven treadmill for 3-4 days, $5 \mathrm{~min} / \mathrm{day}$, on a $10 \%$ grade. The running speed was initially $15 \mathrm{~m} / \mathrm{min}$ and was gradually increased to $25 \mathrm{~m} / \mathrm{min}$ within 2 days. Following the familiarization, all animals performed an incremental test to exhaustion on a treadmill inclined to $15^{\circ}$ [starting at $10 \mathrm{~m} / \mathrm{min}$ with increments of $3 \mathrm{~m} / \mathrm{min}$ every $2 \mathrm{~min}$, (18)]. Exhaustion was defined as the inability of the rats to run on the treadmill despite small electric shocks and an inability to upright themselves when placed on their backs.

Rats were randomly assigned to one of the following three groups: control (CON, $n=10)$, high-intensity training supplemented with a placebo (PLA-T, $n=9$ ), or high-intensity training supplemented with $\mathrm{NaHCO}_{3}(\mathrm{ALK}-\mathrm{T}, n=9)$. Forty-eight hours after the last exercise, all rats were killed by cervical dislocation. Portions of soleus and extensor digitorum longus (EDL) were quickly removed, frozen in liquid nitrogen, and stored at $-80^{\circ} \mathrm{C}$ until biochemical analysis and Western blotting.

\section{Training Intervention}

As shown in Table 1, training consisted of six (1st week) to 12 (5th week) 2-min intervals (interspersed with $1 \mathrm{~min}$ of rest) performed 5 times/wk for $5 \mathrm{wk}$. The intensity of the intervals was initially set at $80 \%$ of the peak speed reached for each rat during the incremental test and was increased by $10 \%$ each week.

\section{Supplementation}

Thirty minutes prior to each training session, PLA-T received water, whereas ALK-T received $\mathrm{NaHCO}_{3}(0.05 \mathrm{mg} / \mathrm{kg}$ body mass $)$
Table 1. Summary of the training performed by both training groups during the experimental period

\begin{tabular}{lrrrrr}
\hline \multicolumn{1}{c}{ Training Weeks } & 1 & 2 & 3 & 4 & 5 \\
\hline $\begin{array}{l}\text { No. of intervals/training session } \\
\text { Mean speed/wk, m/min }\end{array}$ & 6 & 7 & 9 & 10 & 12 \\
$\quad \begin{array}{l}\text { Minimal speed at beginning of training } \\
\quad \text { session, m/min }\end{array}$ & 28 & 28 & 31 & 31 & 37 \\
$\begin{array}{l}\text { Maximal speed reached during training } \\
\quad \text { session, m/min }\end{array}$ & 37 & 40 & 43 & 49 & 52 \\
Mean running distance/training session, m & 435 & 489 & 731 & 962 & 1,202 \\
\end{tabular}

administered by esophageal catheter. The timing was based on our pilot work, which showed that there was a significant increase in blood $\mathrm{pH}$ 15-30 min after the administration of $\mathrm{NaHCO}_{3}$, which was maintained to a steady state for $\$ 45 \mathrm{~min}$ (Fig. 1). The dose was based on previous work in rats (36) that showed that this dose was able to prevent a significant drop in blood bicarbonate concentration following $60 \mathrm{~min}$ of exhaustive exercise. In addition, our further pilot work demonstrated that the decrease in muscle $\mathrm{pH}$ immediately following a typical training session $(6 \times 2$-min intervals interspersed with $1 \mathrm{~min}$ of rest) was significantly less following the administration of $\mathrm{NaHCO}_{3}$ than without $(6.81 \pm 0.04$ vs. $6.96 \pm 0.04 \mathrm{pH}$ units, $P<0.05)$.

\section{Skeletal Muscle Analysis}

Citrate synthase and 6-phosphofructokinase activities. Homogenates for citrate synthase (CS) activity were prepared in buffer [mM: sucrose 210, EGTA 2, NaCl 40, HEPES 30, EDTA 5, and phenylmethylsulfonyl fluoride $2(\mathrm{pH} 7.4)$ ] and stored at $-80^{\circ} \mathrm{C}$. CS activity was assayed by a spectrophotometric method according to Srere (37). Changes in absorbance were recorded for $3 \mathrm{~min}$ at $412 \mathrm{~nm}$ and at $25^{\circ} \mathrm{C}$. Phosphofructokinase (PFK) activity was determined on fresh soleus and EDL homogenates according to the method of Opie and Newsholme (32) and followed the rate of decrease in NADH at 340 $\mathrm{nm}$ and at $25^{\circ} \mathrm{C}$. Muscle protein concentrations were determined in duplicate by Bradford assay with BSA as standard (Bio-Rad, Ivrysur-Seine, France).

Muscle buffer capacity. Resting muscle samples (45-50 mg) were homogenized on ice for $2 \mathrm{~min}$ in a solution containing sodium fluoride $(10 \mathrm{mM})$ at a dilution of $25 \mathrm{ul}$ of homogenizing solution per mg wet wt muscle (see Ref. 29). The muscle homogenate was then placed in a circulating water bath at $37^{\circ} \mathrm{C}$ for $5 \mathrm{~min}$ prior to and during the measurement of $\mathrm{pH}$. The $\mathrm{pH}$ measurements were made with a microelectrode (MI-415; Microelectrodes, Bedford, $\mathrm{NH}$ ) connected to a $\mathrm{pH}$ meter (SA 520; Orion Research, Cambridge, MA). After initial pH measurement, muscle homogenates were adjusted to a $\mathrm{pH}$ of $>7.2$ with a sodium hydroxide solution $(0.1 \mathrm{M})$ and then titrated to a $\mathrm{pH}$ of ?6.2 by the serial addition of $10 \mu \mathrm{l}$ of hydrochloric acid $(0.01 \mathrm{M})$. From the fitted titration trendline, the number of moles of $\mathrm{H}^{+}(\mathrm{per} g$ of wet muscle) required to change the $\mathrm{pH}$ from 7.1 to 6.5 was interpolated. This value was then normalized to the whole $\mathrm{pH}$ unit for final display as micromoles $\mathrm{H}^{+}$per gram wet muscle per unit $\mathrm{pH}$ $\left(\mu \mathrm{mol} \mathrm{H} \mathrm{H}^{+} \cdot \mathrm{g}\right.$ muscle wet $\left.\mathrm{wt}^{-1} \cdot \mathrm{pH}^{-1}\right)$ and determined as the rat's $\left[3 m_{\text {in vitro }}\right.$.

\section{Transport Protein Expression}

Sample preparation for Western blotting. Proteins were isolated from muscles for Western blotting by a method previously described by McCullagh et al. (30) and previously used in our laboratory (39). Muscle protein concentrations were determined in duplicate by bicinchoninic acid assay (Pierce, Interchim, Montluçon, France) with the use of BSA as a standard.

Western blotting of MCT1, MCT4, and NBC. MCT and NBC contents were determined using previously described methods (39). 


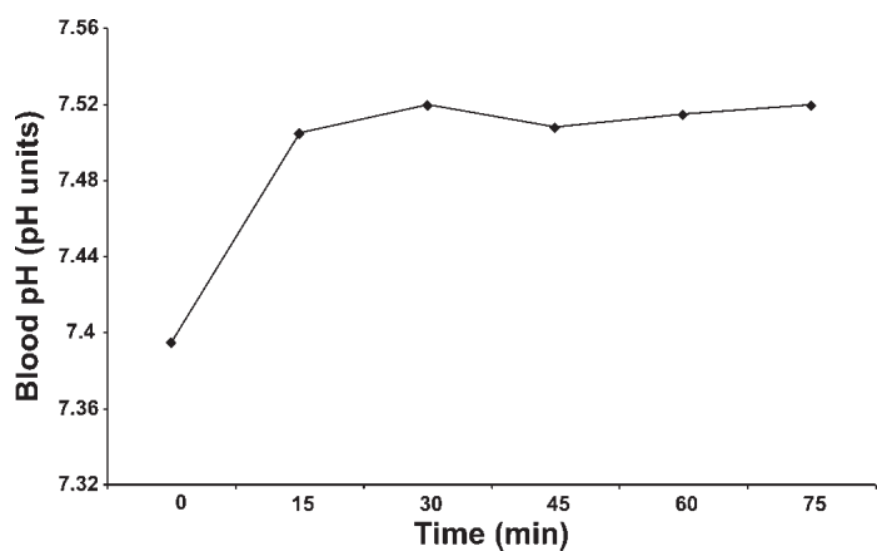

Fig. 1. Evolution of blood $\mathrm{pH}$ after administration of sodium bicarbonate $\left(\mathrm{NaHCO}_{3}\right)$ by esophageal catheter.

Affinity polyclonal antibodies directed against the carboxy terminus of rat MCT1 and MCT4 were produced with the synthetic peptide C-Ahx-PLQNSSGDPAEEESPV-OH (aa 478-494) for MCT1 and C-Ahx-LREVEHFLKAEPEKNG-OH (aa 440-455) for MCT4, like the sequences used by Butz et al. (12), McCullagh et al. (30), and Wilson et al. (41). Such peptides were linked with their cystein residue at KLH EDC-activated carrier protein according to a protocol recommended by the manufacturer (Pierce) and the resulting solutions injected as specific antigens for immunization of New Zealand white rabbits. Western blots were probed with these antibodies and Chemicon International (Temecula, CA) antibodies (rabbit anti-MCT1: AB3540P; rabbit anti-MCT4: AB3314P). Polyclonal antibodies yielded a single band on a Western blot that corresponded to $43 \mathrm{kDa}$, consistent with the molecular mass reported earlier (30). Antibody specificities were confirmed in preliminary experiments in which the peptides blocked the detection of MCT1 and MCT4 (39). To determine NBC expression, we voluntarily chose an NBC antibody against the NBC isoform, which seemed to be related to the kidney/pancreas/ heart isoform NBC1 (150 kDa). Indeed, Kristensen et al. (26) have pointed out that this isoform was clearly present in different rat skeletal muscles, but without any correlation to fiber type. In addition, an NBC construct derived from the kidney corresponding to this isoform was also used by Becker and colleagues $(3,4)$ in their in vitro studies to demonstrate the functional cooperation between NBC and MCT1. The NBC content was quantified by isoform-nonspecific NBC antibodies as previously used by Juel et al. (25) and Kristensen et al. (26) in skeletal muscle (NBC antibody, AF04017; Chemicon International 3212). These NBC antibodies were produced to a sequence (rat kidney NBC amino acids 338-391) with a high degree of homology to most NBC isoforms that have been cloned. Preliminary experiments also confirmed the specificity, and the band was detected at $140-150 \mathrm{kDa}$.

MCT1, MCT4, and NBC expression were detected by ECL (Biomax MR films; Kodak, Reuil-Malmaison, France). Films were developed and processed using a Hyperprocessor, RNP 1700 (Amersham, Les Ulis, France). MCT1 and MCT4 protein band densities were determined by scanning the blots on a scanner (AGFA Duo Scan T1200) and then analyzing with Scion Image software (Scion, Frederick, MD). Results were expressed in arbitrary optical density units as previously used (39).

\section{Statistical Analysis}

Descriptive statistics are expressed as means \pm SE One-way ANOVA was performed to compare the effects of high-intensity training (comparison with $\mathrm{CON}$ group) and the effects of $\mathrm{NaHCO}_{3}$ administration (PLA-T vs. ALK-T). Individual relationships between variables were studied by means of linear regressions. Statview software was used for all tests, and the level of significance was set at $P<0.05$.

\section{RESULTS}

\section{Body Mass}

The mean body mass of the three groups of animals before training was $235 \pm 17 \mathrm{~g}$. After 5 wk of treadmill training, the body masses of the PLA-T and ALK-T rats were $380 \pm 8$ and $367 \pm 8 \mathrm{~g}$, respectively, with no significant difference between groups. After 5 wk of caged living, the control rats weighed significantly more than both training groups $(428 \pm 7 \mathrm{~g}$, $P<0.05)$.

\section{Effects of Training on Changes to CS and PFK Enzyme Activities and Buffer Capacity}

Muscle enzyme activities. As shown in Table 2, compared with CON, the activity of CS in the trained groups was $+16 \%$ higher for PLA-T $(P=0.18)$ and $+23 \%$ higher for ALK-T $(P<0.05)$ in the soleus, with no significant change in the EDL. Furthermore, CS activity was significantly higher in ALK-T than in PLA-T in the soleus $(P<0.05)$. There were also significant changes in PFK activity $(P<0.05)$ in both trained groups $(+48 \%$ for PLA-T and $+45 \%$ for ALK-T) only in the soleus, without change in the EDL after training.

Muscle buffer capacity. As reported in Table 3, muscle buffer capacity significantly increased after both training conditions (placebo and alkalosis) in both the soleus $(P<0.01$, $+41 \%$ for PLA-T and $+40 \%$ for ALK-T) and in the EDL $(P<$ $0.05,+27 \%$ for PLA-T and $+26 \%$ for ALK-T).

\section{Membrane Transport Proteins of Rat Skeletal Muscles}

Skeletal muscle and membrane protein contents. As reported in Table 4, total skeletal muscle and membrane protein contents did not increase after training in PLA-T and ALK-T compared with CON $(P>0.05)$.

MCT1 relative abundance. After training, MCT1 relative abundance in the soleus was significantly greater in the PLA-T $(+30 \%)$ and ALK-T $(+23 \%)$ groups compared with CON $(P<0.05)$, with no significant difference between the two training groups (Fig. 2A). There were no significant differences between the three groups for MCT1 relative abundance in the EDL (Fig. 2B).

Table 2. CS and PFK activity

\begin{tabular}{lccc}
\hline \hline & CON & PLA-T & ALK-T \\
& & & \\
\hline CS activity, $\mu \mathrm{mol} / \mathrm{min}$ & & & \\
$\quad$ SOL & $42.9 \pm 1.6$ & $49.7 \pm 3.4$ & $52.9 \pm 3.2^{*} \dagger$ \\
$\quad$ EDL & $34.1 \pm 3.2$ & $32.4 \pm 3.3$ & $28.6 \pm 3.3$ \\
PFK activity, $\mu \mathrm{mol} \cdot \mathrm{min}^{-1} \cdot \mathrm{mg}$ & & & \\
$\quad$ protein & & & \\
$\quad$ SOL & & & \\
EDL & $9.4 \pm 1.1$ & $13.9 \pm 1.0^{*}$ & $13.6 \pm 1.5^{*}$ \\
& $45.0 \pm 4.3$ & $49.2 \pm 7.4$ & $47.2 \pm 7.4$ \\
\hline
\end{tabular}

Mean values \pm SE for citrate synthase and phosphofructokinase activities in soleus (SOL) and extensor digitorum longus (EDL) muscles in control rats $(\mathrm{CON})$ and trained rats, following the ingestion of either $\mathrm{NaHCO}_{3}(\mathrm{ALK}-\mathrm{T})$ or a placebo (PLA-T). CS, citrate synthase; PFK, phosphofructokinase. ${ }^{*} P<$ 0.05 , significantly different from $\mathrm{CON} ; \uparrow P<0.05$, significantly different from PLA-T. 
Table 3. Muscle buffer capacity

\begin{tabular}{lccc}
\hline \hline & CON & PLA-T & ALK-T \\
\hline $\begin{array}{l}\text { Muscle buffer capacity, } \\
\mu \mathrm{mol} \cdot \mathrm{g}^{-1} \cdot \mathrm{pH}^{-1}\end{array}$ & & & \\
$\quad \mathrm{SOL}$ & $32.9 \pm 1.0$ & $46.4 \pm 2.8^{*}$ & $46.1 \pm 2.0^{*}$ \\
$\mathrm{EDL}$ & $44.0 \pm 1.4$ & $56.1 \pm 3.1^{*}$ & $55.5 \pm 3.9^{*}$
\end{tabular}

Mean values \pm SE for muscle buffer capacity in SOL and EDL muscles in CON and trained rats, following ALK-T or PLA-T. $* P<0.05$, significantly different from CON.

MCT4 relative abundance. MCT4 relative abundance in the soleus was significantly greater in ALK-T $(+115 \%, P<0.01)$ than both PLA-T and CON (Fig. 2A). Although a trend was apparent, there was no significant difference between PLA-T $(+35 \%)$ and $\mathrm{CON}(P=0.30)$. There were no significant differences between the three groups for MCT4 relative abundance in the EDL (Fig. 2B).

$N B C$ relative abundance. $\mathrm{NBC}$ relative abundance in the soleus was significantly greater in both groups $(+85 \%$ for PLA-T and $+60 \%$ for ALK-T) compared with CON, with no significant difference between the two training groups (Fig. $2 A)$. There were no significant differences between the three groups for NBC relative abundance in the EDL (Fig. $2 B$ ).

Relationships between parameters. A positive relationship was observed between MCT1 and NBC $(r=0.50, P<0.05$; Fig. 3), whereas there was no significant relationship between MCT4 and NBC $(r=0.31, P>0.05)$. Furthermore, MCT1 and CS activity were positively correlated in the soleus $(r=$ $0.57, P<0.05)$.

\section{DISCUSSION}

We have observed typical increases in MCT1 and MCT4 relative abundance in response to high-intensity training. This is the first report, however, to demonstrate that NBC relative abundance can also be increased by training. Changes in MCT1, MCT4, and NBC were observed in the soleus only (i.e., a highly oxidative muscle) and not in the EDL (i.e., a highly glycolytic muscle). In contrast, high-intensity training significantly altered the in vitro muscle buffer capacity in both types of muscle. A second novel finding of the present study was that inducing metabolic alkalosis during training was associated with a significantly greater increase in MCT4 relative abundance in the soleus but did not affect other parameters in either muscle. Although there was no relationship between MCT4 and NBC relative abundance, MCT1 and NBC relative abun-

Table 4. Protein content

\begin{tabular}{lccc}
\hline \hline & CON & PLA-T & ALK-T \\
\hline $\begin{array}{l}\text { Total skeletal muscle protein content, } \\
\text { mg/ml }\end{array}$ & & & \\
$\quad$ SOL & $1.5 \pm 0.06$ & $1.3 \pm 0.05$ & $1.3 \pm 0.13$ \\
EDL & $1.2 \pm 0.05$ & $1.1 \pm 0.14$ & $1.1 \pm 0.13$ \\
Muscle membrane protein content, & & & \\
$\quad$ mg/ml & & & \\
SOL & $4.8 \pm 0.4$ & $4.9 \pm 0.5$ & $4.3 \pm 0.3$ \\
EDL & $6.9 \pm 0.7$ & $7.1 \pm 0.8$ & $6.6 \pm 0.7$ \\
\hline
\end{tabular}

Mean values \pm SE for 1 ) total skeletal muscle protein content and 2) membrane protein content in SOL and EDL in CON and trained rats following ALK-T or PLA-T. $P>0.05$, significantly different from CON.

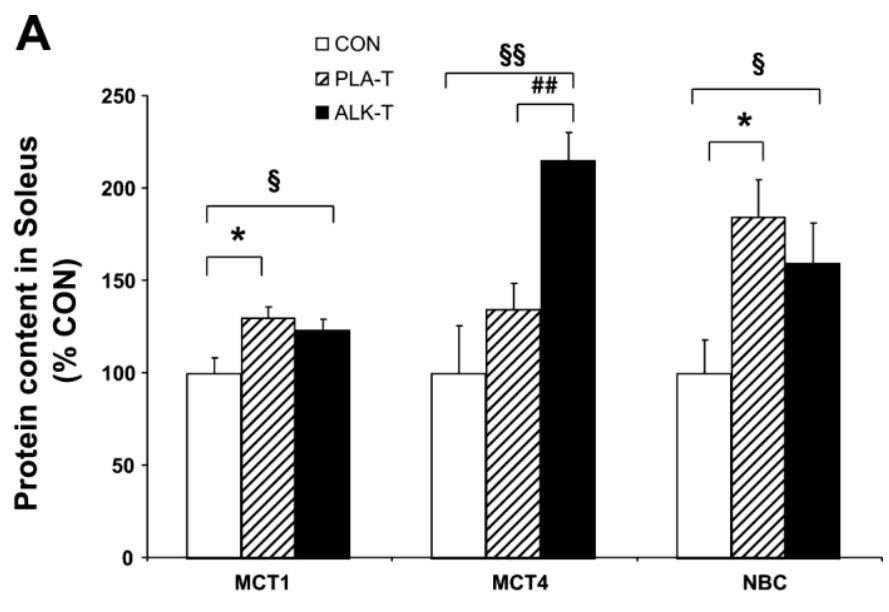

B

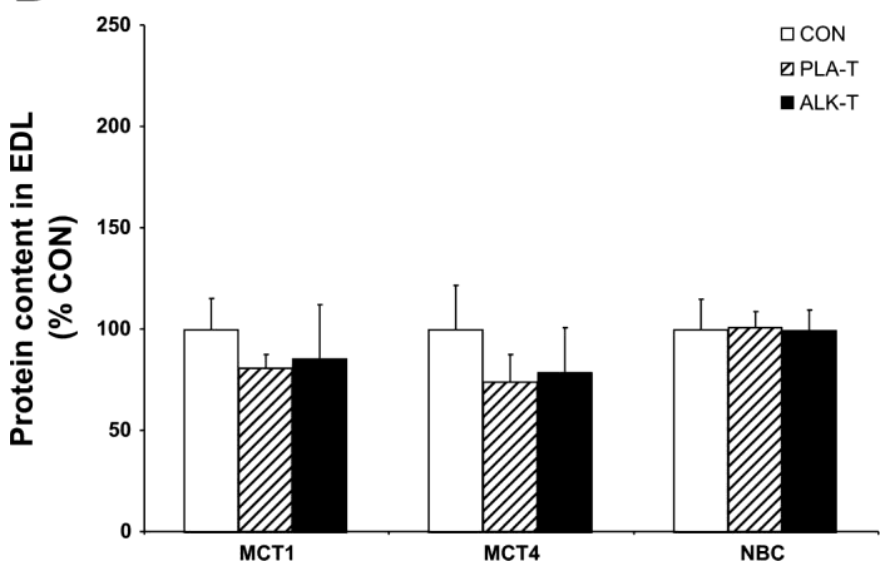

Fig. 2. Monocarboxylate transporter (MCT)1, MCT4, and $\mathrm{NaHCO}_{3}$ transporter $(\mathrm{NBC})$ relative abundance in muscle membrane preparations of trained rats following $5 \mathrm{wk}$ of high-intensity interval training with the administration of either $\mathrm{NaHCO}_{3}$ (ALK-T) or a placebo (PLA-T) prior to training session (in $\%$ of control values) in the soleus muscle $(A)$ and the extensor digitorum longus muscle (EDL; $B$ ). ${ }^{*} P<0.05$, significant difference between control $(\mathrm{CON})$ and PLA-T; $\$ P<0.05$, significant difference between CON and ALK-T; $\$ \S P<0.01$, significant difference between CON and ALK-T; \#\#P< 0.01 , significant difference between PLA-T and ALK-T.

dance were positively related in oxidative, but not glycolytic, muscle.

\section{Training Adaptations}

In the present study, MCT1 and MCT4 relative abundance were higher after training than in the control condition in oxidative muscle. These findings are in accordance with observations in other studies that have demonstrated that a period of training can increase the density of MCT1 $(14,24,34)$ and MCT4 (34) proteins in skeletal muscle. Despite not reaching statistical significance, the percentage increase in MCT4 was similar to that reported in previous studies $(24,31,34)$. The lack of signifi in the present study can partly be attributed to the greater variability in the MCT4 response (i.e., low statistical power), which is again typical of previous research $(9,14)$.

In the present study, we confirm, via Western blotting, that NBC is present in the membranes of both oxidative and glycolytic skeletal muscle $(25,26)$. For the first time, however, 


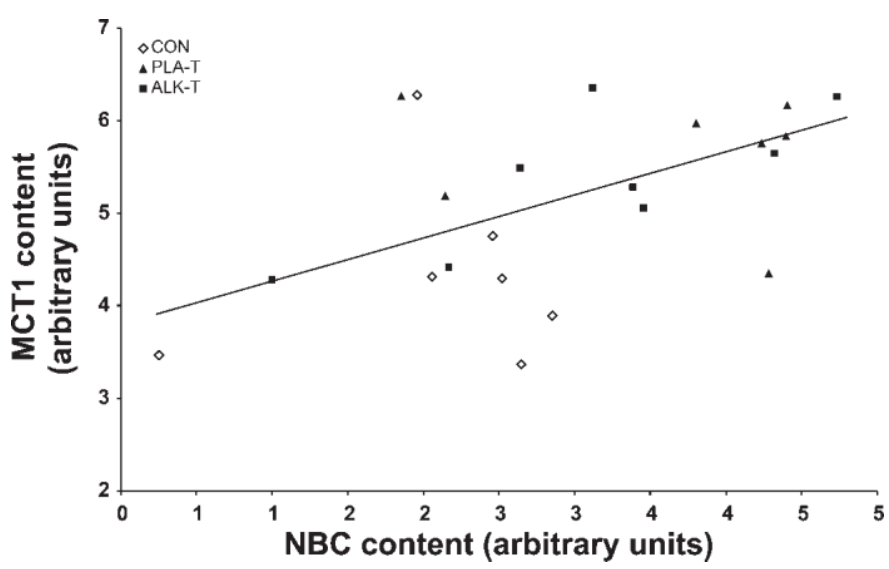

Fig. 3. Relationship between the relative abundance of MCT1 and NBC in the soleus muscle membrane preparations of $\mathrm{CON}$ and trained rats following $5 \mathrm{wk}$ of high-intensity interval training with ALK-T or PLA-T prior to each training session. $(r=0.50, P<0.05)$.

we have also shown that, like MCT1 and MCT4, NBC relative abundance can be increased by exercise training. Similar to previous results for MCT1 (2), we report that NBC also responds with a fiber-type specificity to high-intensity training (i.e., changes were observed in the soleus but not the EDL). Thus, we have shown that, like other membrane transport proteins that are involved in muscle $\mathrm{pH}$ regulation [i.e., MCT1 $(14,24), \operatorname{MCT} 4(24,31)$, and $\operatorname{NHE}(24,31)], \mathrm{NBC}$ is sensitive to chronic contractile activity in rat skeletal muscle.

The lack of change in either MCT1, MCT4, or NBC relative abundance in the EDL suggests that these proteins respond in a fiber-specific manner. One can suggest that potential increases in any of the proteins that we measured could have been underestimated due to an increase in total protein and/or total membrane protein contents. However, no change in protein contents in EDL and soleus was observed after training (Table 4), confirming the fiber-type specific response of these proteins. Furthermore, our results are consistent with previous research (2) that has reported greater training-induced increases in MCT1 in red than in white muscle in rats. Furthermore, Bonen (7) has demonstrated the same fiber-specific response of MCT1 to chronic muscle stimulation. These muscle-specific changes are unlikely to be due to less recruitment of the EDL during treadmill running exercise, as our pilot work demonstrated significant decreases in muscle $\mathrm{pH}$ in the EDL muscle after a typical exercise session. We also reported a significant increase in $\left[3 m_{\text {in }}\right.$ vitro in both the EDL and the soleus (Table 3).

\section{Regulation of NBC, MCT1, and MCT4 Expression}

Because MCT1, MCT4, and NBC all contribute to $\mathrm{pH}$ regulation, it might be predicted that $\mathrm{H}^{+}$accumulation during training would be an important factor stimulating the synthesis of these proteins. Support for this idea is provided by previous research $(13,24,31)$ that has shown that increases in NHE content (another important $\mathrm{H}^{+}$transporter) tend to be greater in response to training that leads to a significant decrease in $\mathrm{pH}$. Therefore, to investigate the role of $\mathrm{H}^{+}$accumulation on MCT1, MCT4, and NBC adaptations to training in rats, we used sodium bicarbonate to experimentally manipulate the intracellular $\mathrm{H}^{+}$concentration during high-intensity training.
As demonstrated by our pilot work, muscle proton accumulation differed in our two training groups (PLA-T > ALK-T).

In contrast to our hypothesis, the present results indicate that performing the same training with different $\mathrm{H}^{+}$accumulation did not alter the training-induced improvements in MCT1 (+23\% for ALK-T vs. $+30 \%$ for PLA-T, $P>0.05)$. This fi is supported by the results of a recent study (31) demonstrating that increases in MCT1 in humans were not signifi different between two different training programs that resulted in signifi different changes in muscle $\mathrm{pH}$ (7.06 vs. 6.98). The observation that increases in NBC in the present study were also not signifi different between conditions $(+60 \%$ for ALK-T vs. $+85 \%$ for PLA-T, $P>0.05)$ may be related to the functional cooperation that has been identifi between MCT1 and NBC (3). Because an adequate contraction stimulus appears to be required to increase MCT1 density (2), we cannot exclude the possibility that a minimum accumulation of $\mathrm{H}^{+}$is required to stimulate MCT1 and NBC synthesis. However, it appears that the degree of $\mathrm{H}^{+}$accumulation is not the main factor stimulating further synthesis of these proteins. Recently, Hashimoto et al. (17) have demonstrated that lactate anion could be a signaling molecule to regulate MCT1 expression, but not MCT4, in L6 cells.

A novel finding of the present study was that the group that ingested sodium bicarbonate prior to training had a significantly greater increase in MCT4 relative abundance in the soleus than the placebo training group $(+115$ vs. $+35 \%, P<$ 0.01; Fig. 2). Because control rats did not receive bicarbonate ingestion, we cannot exclude the possible effects of bicarbonate ingestion alone. However, since MCT4 content tended to increase in PLA-T, this suggests that the greater improvements in the ALK-T were due to the combined effects of training and sodium bicarbonate ingestion, possibly mediated via a reduced $\mathrm{H}^{+}$accumulation during training. Although sodium bicarbonate ingestion has also been reported to increase muscle lactate accumulation (27), this appears unlikely to explain the differences in MCT4, as Mohr et al. (31) have reported no significant differences in MCT4 following two training programs that elicited very different muscle lactate values.

The mechanisms responsible for the greater improvements in MCT4 in ALK-T, when compared with PLA-T, cannot be determined from the present study. However, flux through a transport system during high-intensity exercise may provide a crucial stimulus for adaptation (24), and hydrogen ion efflux out of muscle cells has been reported to be enhanced by a greater extracellular buffer concentration (28). Therefore, it is possible that, in the present study, alkalosis induced a greater release of lactate and protons from working muscle (27) and that this stimulated greater MCT4 expression. It is difficult to speculate as to why this effect was seen only in the soleus (a highly oxidative muscle). However, it has been reported (7) that MCT4 content is greater in glycolytic fibers, and this may make it difficult to further elevate MCT4 content in such fibers (e.g., the EDL). Although sodium bicarbonate ingestion did not influence training-induced adaptations in MCT1 (or NBC), this is in accordance with previous research $(10,17,24)$ demonstrating that transporters are regulated in an isoform-specific manner. 


\section{Relationship Between MCT1 and NBC}

A further novel finding was the moderate, positive relationship between MCT1 and NBC relative abundance in oxidative, but not glycolytic, muscle. A greater MCT1 content has previously been shown (30) to be related to an increase in the transport of lactate (and $\mathrm{H}^{+}$) into the muscle. It may, therefore, be important that increases in MCT1 are accompanied by increases in NBC, as observed in the soleus in the present study, to help suppress the buildup of extracellular $\mathrm{H}^{+}$and to maintain the $\mathrm{pH}$ gradient that is required for continued MCT1 activity (22). This hypothesis is supported by previous in vitro research that has shown that, when MCT1 is coexpressed with $\mathrm{NBC} 1$ in oocytes, lactate transport is enhanced (3). In this way, MCT1 and NBC could cooperate to facilitate lactate uptake in oxidative skeletal muscle during high-intensity exercise and recovery.

The moderate correlation coefficient between NBC and MCT1 relative abundance $(r=0.50, P<0.05)$, however, suggests that other parameters are also likely to be involved in the regulation of lactate transport during high-intensity exercise and recovery. Of crucial importance to this process is the distribution and activity of carbonic anhydrase (CA), both in skeletal muscle and in the extracellular space, which help to establish a fast,equilibrium between carbonic acid and $\mathrm{CO}_{2}$, $\mathrm{HCO}_{3}$, and $\mathrm{H}^{+}$(16). In addition, it has been reported that carbonic anhydrase IV, which is the sarcolemmal CA (sCA) isoform (16), has an essential interaction for full NBC1 activity (1). Recently, sCA has been shown to facilitate lactic acid transport in rat skeletal muscles (40). In consequence, although we have not demonstrated the colocalization of MCT1 and $\mathrm{NBC}$, our results and those of the literature are in favor of a combination of NBC, MCT1, and SCA that may help to make the lactate shuttle more efficient between blood and muscle and to improve intracellular $\mathrm{pH}$ regulation, particularly after training. Surprisingly, no data are currently available concerning the relationship between MCT4 and either NBC or sCA. We did not, however, find a significant relationship between MCT4 and NBC relative abundance in the present study.

\section{Conclusions}

In conclusion, the present study has shown that MCT1, MCT4, and NBC undergo fiber-specific adaptive changes in response to high-intensity training and that NBC relative abundance is moderately related to MCT1 expression in oxidative, but not glycolytic, muscle. In consequence, we can speculate that lactate transport may be facilitated by NBC in oxidative skeletal muscle. Furthermore, although MCT4 content tended to increase with high-intensity training, alkalosis induced prior to each training session resulted in a significantly greater increase in MCT4 relative abundance in the soleus muscle, without any effect on MCT1 and NBC content.

\section{ACKNOWLEDGMENTS}

We thank Prof. Dominique Mornet and Gerald Hugon for the production of MCT1 and MCT4 antibodies.

\section{REFERENCES}

1. Alvarez BV, Loiselle FB, Supuran CT, Schwartz GJ, Casey JR. Direct extracellular interaction between carbonic anhydrase IV and the human
NBC1 sodium/bicarbonate co-transporter. Biochemistry 42: 12321-12329, 2003.

2. Baker SK, McCullagh KJ, Bonen A. Training intensity-dependent and tissue-specific increases in lactate uptake and MCT-1 in heart and muscle. J Appl Physiol 84: 987-994, 1998.

3. Becker HM, Broer S, Deitmer JW. Facilitated lactate transport by MCT1 when coexpressed with the sodium bicarbonate cotransporter (NBC) in Xenopus oocytes. Biophys $J$ 86: 235-247, 2004.

4. Becker HM, Deitmer JW. Voltage dependence of H+ buffering mediated by sodium bicarbonate cotransport expressed in Xenopus oocytes. J Biol Chem 279: 28057-28062, 2004.

5. Bickham DC, Bentley DJ, Le Rossignol PF, Cameron-Smith D. The effects of short-term sprint training on MCT expression in moderately endurance-trained runners. Eur J Appl Physiol 96: 636-643, 2006.

6. Bonen A. The expression of lactate transporters (MCT1 and MCT4) in heart and muscle. Eur J Appl Physiol 86: 6-11, 2001.

7. Bonen A. Lactate transporters (MCT proteins) in heart and skeletal muscles. Med Sci Sports Exerc 32: 778-789, 2000.

8. Bonen A, Heynen M, Hatta H. Distribution of monocarboxylate transporters MCT1-MCT8 in rat tissues and human skeletal muscle. Appl Physiol Nutr Metab 31: 31-39, 2006.

9. Bonen A, McCullagh KJ, Putman CT, Hultman E, Jones NL, Heigenhauser GJ. Short-term training increases human muscle MCT1 and femoral venous lactate in relation to muscle lactate. Am J Physiol Endocrinol Metab 274: E102-E107, 1998.

10. Bonen A, Tonouchi M, Miskovic D, Heddle C, Heikkila JJ, Halestrap AP. Isoform-specific regulation of the lactate transporters MCT1 and MCT4 by contractile activity. Am J Physiol Endocrinol Metab 279: E1131-E1138, 2000.

11. Brooks GA. Intra- and extra-cellular lactate shuttles. Med Sci Sports Exerc 32: 790-799, 2000.

12. Butz CE, McClelland GB, Brooks GA. MCT1 confirmed in rat striated muscle mitochondria. J Appl Physiol 97: 1059-1066, 2004.

13. Dela F, Holten M, Juel C. Effect of resistance training on Na,K pump and $\mathrm{Na}+/ \mathrm{H}+$ exchange protein densities in muscle from control and patients with type 2 diabetes. Pflügers Arch 447: 928-933, 2004.

14. Dubouchaud H, Butterfield GE, Wolfel EE, Bergman BC, Brooks GA. Endurance training, expression, and physiology of LDH, MCT1, and MCT4 in human skeletal muscle. Am J Physiol Endocrinol Metab 278: E571-E579, 2000.

15. Edge J, Bishop D, Goodman C. Effects of chronic $\mathrm{NaHCO}_{3}$ ingestion during interval training on changes to muscle buffer capacity, metabolism, and short-term endurance performance. J Appl Physiol 101: 918-925, 2006.

16. Geers C, Gros G. Carbon dioxide transport and carbonic anhydrase in blood and muscle. Physiol Rev 80: 681-715, 2000.

17. Hashimoto T, Hussien R, Oommen S, Gohil K, Brooks GA. Lactate sensitive transcription factor network in L6 cells: activation of MCT1 and mitochondrial biogenesis. FASEB J. 21: 2602-2612, 2007.

18. Henderson KK, Wagner H, Favret F, Britton SL, Koch LG, Wagner PD, Gonzalez NC. Determinants of maximal $\mathrm{O}_{2}$ uptake in rats selectively bred for endurance running capacity. J Appl Physiol 93: 1265-1274, 2002.

19. Juel C. Expression of the $\mathrm{Na}(+) / \mathrm{H}(+)$ exchanger isoform NHE1 in rat skeletal muscle and effect of training. Acta Physiol Scand 170: 59-63, 2000.

20. Juel C. Muscle lactate transport studied in sarcolemmal giant vesicles. Biochim Biophys Acta 1065: 15-20, 1991.

21. Juel C. Training-induced changes in membrane transport proteins of human skeletal muscle. Eur J Appl Physiol 96: 627-635, 2006.

22. Juel C, Halestrap AP. Lactate transport in skeletal muscle-role and regulation of the monocarboxylate transporter. J Physiol 517: 633-642, 1999.

23. Juel C, Holten MK, Dela F. Effects of strength training on muscle lactate release and MCT1 and MCT4 content in healthy and type 2 diabetic humans. J Physiol 556: 297-304, 2004.

24. Juel C, Klarskov C, Nielsen JJ, Krustrup P, Mohr M, Bangsbo J. Effect of high-intensity intermittent training on lactate and $\mathrm{H}^{+}$release from human skeletal muscle. Am J Physiol Endocrinol Metab 286: E245-E251, 2004

25. Juel C, Lundby C, Sander M, Calbet JA, Hall G. Human skeletal muscle and erythrocyte proteins involved in acid-base homeostasis: adaptations to chronic hypoxia. J Physiol 548: 639-648, 2003. 
26. Kristensen JM, Kristensen M, Juel C. Expression of $\mathrm{Na}+\mathrm{HCO} 3-\mathrm{co}-$ transporter proteins (NBCs) in rat and human skeletal muscle. Acta Physiol Scand 182: 69-76, 2004.

27. Lindinger MI, Heigenhauser GJ, Spriet LL. Effects of alkalosis on muscle ions at rest and with intense exercise. Can J Physiol Pharmacol 68: 820-829, 1990 .

28. Mainwood GW, Worsley-Brown P. The effects of extracellular $\mathrm{pH}$ and buffer concentration on the efflux of lactate from frog sartorius muscle. J Physiol 250: 1-22, 1975.

29. Mannion AF, Jakeman PM, Willan PL. Determination of human skeletal muscle buffer value by homogenate technique: methods of measurement. J Appl Physiol 75: 1412-1418, 1993.

30. McCullagh KJ, Poole RC, Halestrap AP, O'Brien M, Bonen A. Role of the lactate transporter (MCT1) in skeletal muscles. Am J Physiol Endocrinol Metab 271: E143-E150, 1996.

31. Mohr M, Krustrup P, Nielsen JJ, Nybo L, Rasmussen MK, Juel C, Bangsbo J. Effect of two different intense training regimens on skeletal muscle ion transport proteins and fatigue development. Am J Physiol Regul Integr Comp Physiol 292: R1594-R1602, 2007.

32. Opie L, Newsholme EA. The activities of fructose 1,6-diphosphatase, phosphofructokinase and phosphoenolpyruvate carboxykinase in white and red muscle. Biochem J 103: 391-399, 1967.

33. Parkhouse WS, McKenzie DC, Hochachka PW, Ovalle WK. Buffering capacity of deproteinized human vastus lateralis muscle. J Appl Physiol 58: 14-17, 1985.
34. Pilegaard H, Domino K, Noland T, Juel C, Hellsten Y, Halestrap AP Bangsbo J. Effect of high-intensity exercise training on lactate $/ \mathrm{H}^{+}$transport capacity in human skeletal muscle. Am J Physiol Endocrinol Metab 276: E255-E261, 1999.

35. Pilegaard H, Terzis G, Halestrap A, Juel C. Distribution of the lactate/ $\mathrm{H}^{+}$transporter isoforms MCT1 and MCT4 in human skeletal muscle. Am J Physiol Endocrinol Metab 276: E843-E848, 1999.

36. Rico H, Aznar L, Hernandez ER, Seco C, Sanchez-Atrio A, Villa LF, Gervas JJ. Effects of potassium bicarbonate supplementation on axial and peripheral bone mass in rats on strenuous treadmill training exercise. Calcif Tissue Int 65: 242-245, 1999.

37. Srere P. Citrate synthase. Methods Enzymol 13: 3-5, 1969.

38. Street D, Nielsen JJ, Bangsbo J, Juel C. Metabolic alkalosis reduces exercise-induced acidosis and potassium accumulation in human skeletal muscle interstitium. J Physiol 566: 481-489, 2005.

39. Thomas C, Perrey S, Lambert K, Hugon G, Mornet D, Mercier J. Monocarboxylate transporters, blood lactate removal after supramaximal exercise, and fatigue indexes in humans. J Appl Physiol 98: 804-809, 2005 .

40. Wetzel P, Hasse A, Papadopoulos S, Voipio J, Kaila K, Gros G. Extracellular carbonic anhydrase activity facilitates lactic acid transport in rat skeletal muscle fibres. J Physiol 531: 743-756, 2001.

41. Wilson MC, Jackson VN, Heddle C, Price NT, Pilegaard H, Juel C, Bonen A, Montgomery I, Hutter OF, Halestrap AP. Lactic acid efflux from white skeletal muscle is catalyzed by the monocarboxylate transporter isoform MCT3. J Biol Chem 273: 15920-15926, 1998. 